\title{
Long-term evaluation of a structured outpatient education programme for intensified insulin therapy in patients with Type 1 diabetes: a 12-year follow-up
}

\author{
J. Plank ${ }^{1}$ G. Köhler ${ }^{1}$ I. Rakovac ${ }^{2}$ B. M. Semlitsch ${ }^{1}$ K. Horvath ${ }^{1}$ G. Bock ${ }^{1}$ B. Kraly ${ }^{1}$ T. R. Pieber ${ }^{1,2}$ \\ ${ }^{1}$ Division of Diabetes and Metabolism, Department of Internal Medicine, Medical University Hospital, Graz, Austria \\ 2 Joanneum Research, Institute of Medical Technologies and Health Management, Graz, Austria
}

\begin{abstract}
Aims/hypothesis. This study was conducted to evaluate the long-term outcome of a structured outpatient diabetes teaching and treatment programme (DTTP) for intensified insulin therapy in patients with Type 1 diabetes, which aims to improve metabolic control without increasing the risk of severe hypoglycaemia.

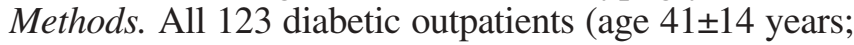

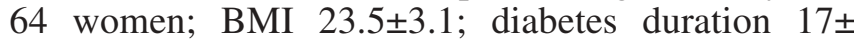
11 years; $\mathrm{HbA}_{1} \mathrm{c} 7.9 \pm 1.6 \% ; 32$ patients with a history of severe hypoglycaemia; 18 with overt nephropathy; 22 with proliferative retinopathy) who participated in the DTTP between June 1989 and June 1990 were invited for follow-up visits after 3, 6 and 12 years.

Results. Out of the 123 patients, 11 died during the follow-up period, two were lost for follow-up, and one was not willing to participate in re-evaluation after

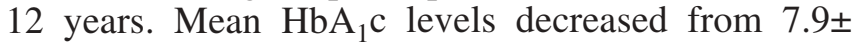

$1.6 \%$ to $7.1 \pm 1.2 \%(p<0.01)$ after 3 years, and were $7.8 \pm 1.5 \%(\mathrm{NS})$ and $7.8 \pm 1.2 \%(\mathrm{NS})$ after 6 and 12 years respectively. Frequency of hypoglycaemia decreased from 0.49 episodes per patient per year to 0.14 after 3 years $(p<0.01), 0.19$ after 6 years $(p<0.01)$ and 0.16 after 12 years $(p<0.01)$. Of the participants, $41 \%$ were able to lower $\mathrm{HbA}_{1} \mathrm{c}$ levels without episodes of severe hypoglycaemia and to maintain this improvement at all follow-up visits over the 12-year period. At follow-up, intensified insulin therapy was carried out by $94 \%$ of the patients.

Conclusions/interpretation. A sustained reduction of the incidence of severe hypoglycaemia was observed in patients with Type 1 diabetes after participation in a structured outpatient DTTP over a 12-year period.

Keywords Cohort study - Complications - Education · Healthcare delivery $\cdot$ Severe hypoglycaemia
Received: 19 March 2004 / Accepted: 10 June 2004

Published online: 17 July 2004

(C) Springer-Verlag 2004

\section{J. Plank (®)}

Division of Diabetes and Metabolism,

Department of Internal Medicine,

Medical University Hospital,

Auenbruggerplatz 15, 8036 Graz, Austria

E-mail: johannes.plank@klinikum-graz.at

Tel.: +43-316-3853270, Fax: +43-316-3854332

Abbreviations: DTTP, diabetes teaching and treatment programme - EDIC, Epidemiology of Diabetes Interventions and Complications · IIT, intensified insulin therapy

\section{Introduction}

Dietary restrictions have a negative impact on the quality of life in patients with diabetes, as the need to ingest fixed amounts of carbohydrates, proteins and fats at prescheduled times interferes with normal flexible daily life in most patients [1]. In patients with

Conflict of interest: This trial was supported in part by the Center of Excellence Project of Novo Nordisk. T. Pieber was a paid consultant for Novo Nordisk, Eli Lilly, and Aventis, and is on the advisory board of Novo Nordisk. I. Rakovac received an educational grant from the Austrian Diabetes Association 2003, sponsored by Novo Nordisk and Aventis. The Research Group performed trials for all three major insulin-producing companies. 
Type 1 diabetes, frequent metabolic self-monitoring and self-adaptation of insulin dosages are necessary to simultaneously liberalise diet and to establish near normoglycaemia. The DCCT [2] has clearly proven that tight blood glucose control and the resulting longterm benefits can be achieved by intensified insulin therapy (IIT) [3]. However, intensification of insulin therapy resulted in a three-fold increase in the incidence of severe hypoglycaemia compared with conventional therapy, and over 7 years of the Epidemiology of Diabetes Interventions and Complications (EDIC) study, the annual follow-up examinations of the DCCT population showed an overall mean $\mathrm{HbA}_{1} \mathrm{c}$ increase to $8.1 \%$ for the intensified treatment group $[2,3,4]$. In the DCCT, insulin dose adjustment was performed by clinicians during frequent outpatient visits, rather than by patients based on recent blood glucose readings. Patient education is a prerequisite for avoiding the major side effect of IIT, hypoglycaemia. In patients undergoing a structured teaching and treatment programme for IIT, this unacceptably high risk was not observed. Reduced risk of severe hypoglycaemia and improved metabolic control after intensification of insulin therapy was demonstrated for a period of up to 6 years $[5,6]$. Moreover, owing to dietary liberalisation, the patients' quality of life was found to be substantially improved in a randomised controlled multicentre trial in the UK using the outpatient teaching programme [7].

In 1989 a structured teaching and treatment programme for Type 1 diabetic patients was implemented as part of the standard care in the diabetes division of the University Hospital in Graz, Austria. To reduce costs of hospitalisation, the inpatient basis of the teaching and treatment programme was adjusted to an outpatient basis for the first time. The effectiveness of this setting after 3 and 6 years has been reported [5, $8]$. The aim of this investigation was to evaluate over a 12-year period the beneficial effects in patients with Type 1 diabetes of participation in a structured 5-day teaching and treatment programme for IIT in an outpatient setting.

\section{Subjects and methods}

Subjects. The study population consisted of patients with Type 1 diabetes with a treatment duration of longer than 12 months who were referred to the diabetes outpatient clinic of the University Hospital in Graz between June 1989 and June 1990 to participate in the structured outpatient diabetes teaching and treatment programme (DTTP). Most patients were referred by their family doctors or general internists in private practice. The main reason for referral was unsatisfactory metabolic control. Some were referred by other hospitals mainly because of existing late complications [5]. Apart from severe physical or mental impairment preventing patients from following the structured 5-day programme, there were no reasons for exclusion, such as the presence of any diabetes-related complications, a long duration of diabetes, older age or a history of severe hypoglycaemia. After 3, 6 and 12 years, all patients were invited to a follow-up examination. The protocol was approved by the local ethics committee and all procedures were carried out in accordance with the Declaration of Helsinki.

The diabetes teaching and treatment programme. The DTTP evaluated in this study was based on programmes developed for patient education in Type 1 diabetic patients $[9,10,11]$ and has been described in detail previously [5]. Briefly, a diabetes nurse educator and a dietician taught a structured course to groups of five to eight outpatients. The objectives of the 5-day teaching and treatment programme were to improve glycaemic control without increasing the risk of severe hypoglycaemia, and to liberalise the diet by matching insulin doses to food choices and actual blood glucose levels. This flexible use of insulin with immediate dosage adjustment is considered a prerequisite for achieving treatment goals. Furthermore, early recognition and immediate treatment of low blood glucose levels, which are mandatory to avoid severe hypoglycaemia, were particularly emphasised throughout the teaching programme.

Study protocol. After the DTTP, all participants were offered ongoing routine care in our outpatient clinic with at least annual consultation. During follow-up visits after 3, 6 and 12 years, aspects of diabetes care were assessed using a clinical assessment and a standardised interview [5]. Severe hypoglycaemia was defined as a hypoglycaemic episode requiring assistance of another person and treatment by intravenous glucose or glucagon injection. At baseline and each follow-up visit, frequency of severe hypoglycaemia was assessed retrospectively for the year before evaluation. $\mathrm{HbA}_{1} \mathrm{c}$ was measured by Diamat HPLC (Bio-Rad, Milan, Italy) at baseline and after 3 and 6 years, and by Hi-Auto $\mathrm{A}_{1} \mathrm{c}$ analyser system HA-8140 (Menarini Diagnostics, Florence, Italy) at the 12-year assessment. Values were converted to correspond with those of the HA-8140 HPLC method (reference range 4.3-5.9; $y=0.95 x-0.02 ; r=0.98$ [12]). Protein concentration was measured in a 24-h urine sample. In cases where a 24-h urine sample was not provided, a first morning sample was used. Records of recent annual ophthalmological examinations were collected at follow-up. A questionnaire with 39 questions on different aspects of diabetes management was used to assess diabetes-related knowledge before and after the training programme and at each follow-up visit.

Statistical analysis. Statistical analyses were performed using generalised estimating equations [13] to account for repeated measures on patients. An unstructured correlation matrix was used in all computations.

In analyses of development over time, the time of followup examination was modelled as a factor. For continuous response variables, the Gaussian model family was used and the difference of means between follow-up and baseline, along with the corresponding $95 \% \mathrm{CI}$, was reported. For binomial response variables (e.g. proportion of patients without daily blood glucose monitoring), the binomial family was used and the odds ratio between follow-up and baseline proportion, along with the corresponding 95\% CI, was reported. For counting variables (e.g. number of episodes of severe hypoglycaemia, number of daily insulin injections), the Poisson family was used and the rate ratio, along with the corresponding $95 \%$ CI, was reported.

Analyses were performed using the $\mathrm{R}$ package for statistical computing [14]. In all computations, a result was considered statistically significant if the $p$ value was less than 0.05 . 


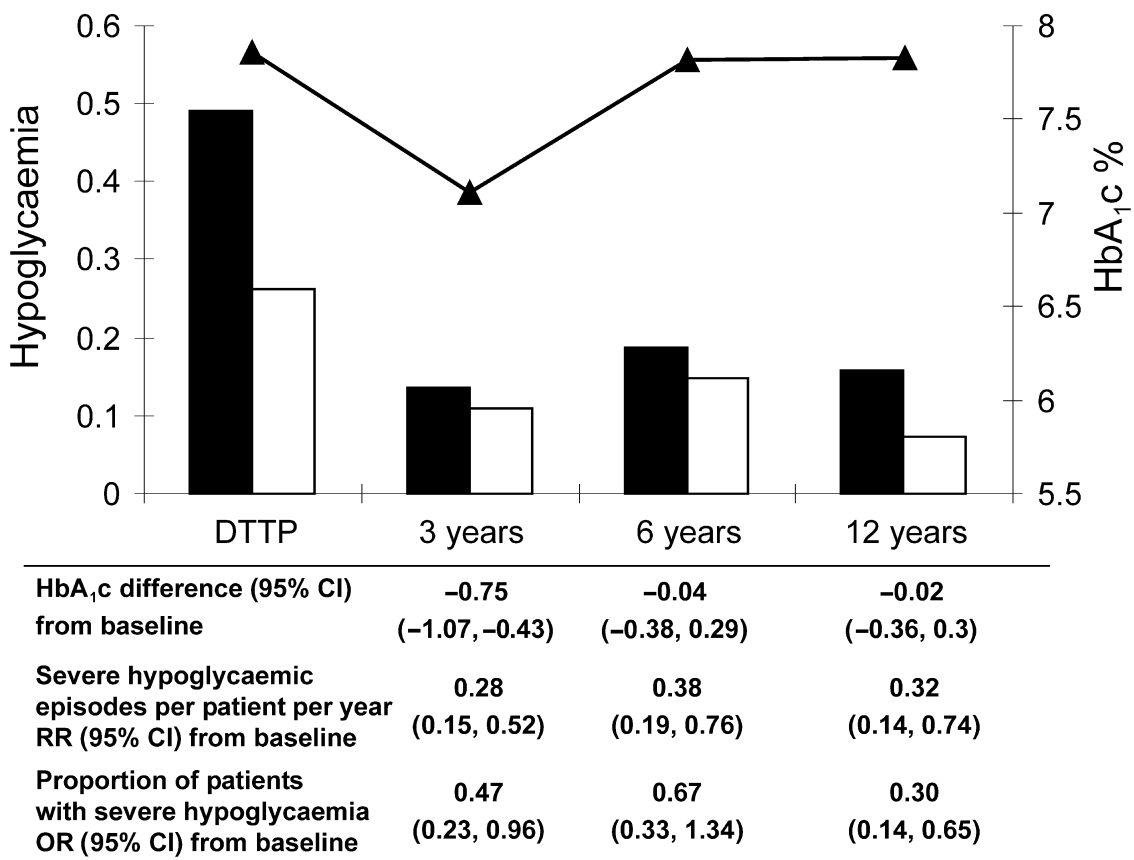

Fig. 1. Metabolic control in 123 patients with Type 1 diabetes after the DTTP. Shown are episodes of severe hypoglycaemia per patient (black) and percentage of patients with at least one episode of severe hypoglycaemia (white) in the preceding year, as well as $\mathrm{HbA}_{1} \mathrm{c}$ levels over the 12 -year period (black triangles). Differences from baseline are also displayed. OR, odds ratio; $\mathrm{RR}$, rate ratio

\section{Results}

Baseline data and participant follow-up. All 123 patients who participated in the DTTP from June 1989 to June 1990 were invited for follow-up visits after 3, 6 and 12 years. Patient characteristics at baseline are displayed in Table 1.

After 3 years, all but two patients, who moved out of central Europe and remained lost for follow-up for the whole observation period, were re-evaluated. After 6 years, an additional nine patients were not willing to participate in the follow-up, and four were unable to take part for reasons such as work commitments. Only one out of these 13 patients was not willing to return for re-examination at the 12-year follow-up. Therefore, data was available for 121, 108 and 120 patients after 3, 6 and 12 years respectively.

Metabolic control. Results of metabolic control are shown in Figure 1. After initiation of IIT during the DTTP, $\mathrm{HbA}_{1} \mathrm{c}$ decreased from $7.9 \pm 1.6 \%$ to $7.1 \pm 1.2 \%$ after 3 years $(p<0.001)$. After 6 years, $\mathrm{HbA}_{1} \mathrm{c}$ increased again to $7.8 \% \pm 1.5 \%(\mathrm{NS})$. At the 12-year assessment, $\mathrm{HbA}_{1} \mathrm{c}$ was $7.8 \pm 1.2 \%$ (NS).

The frequency of severe hypoglycaemia decreased from 0.49 episodes per patient per year before the DTTP to $0.14,0.19$ and 0.16 after 3,6 and 12 years respectively (all $p<0.01$ vs baseline). In the year preceding the DTTP, $32(26 \%)$ patients had a history of

Table 1. Baseline characteristics of the 123 participants of the DTTP

Characteristic

Age (years)

Females $(n)$

Ethnicity: Caucasian (\%)

Duration of diabetes (years)

BMI $\left(\mathrm{kg} / \mathrm{m}^{2}\right)$

$\mathrm{HbA}_{1} \mathrm{c}(\%)$

Severe hypoglycaemia in preceding year $(n)$

Rate ratio, systolic : diastolic $(\mathrm{mm} \mathrm{Hg})$

Advanced retinopathy $(n)$

Overt nephropathy $(n)$

Antihypertensive prescriptions ( $n)$

Unless otherwise stated, data are expressed as means \pm SD

severe hypoglycaemia. In 14 (12\%), 19 (18\%) and $9(8 \%)$ patients, severe hypoglycaemia occurred during the 12 months before re-evaluation at 3,6 and 12 years $(p<0.01$, NS, and $p<0.01)$ respectively.

The primary aim of the DTTP, to improve metabolic control without occurrence of severe hypoglycaemia, was achieved by $69 \%$ of participants at the 3 -year follow-up. The same goal was achieved in $52 \%$ of patients at the 6-year as well as the 3-year followup, and for $41 \%$ this was achieved at the 3-year, 6-year and 12-year follow-up.

Hyperglycaemic emergencies were a rare event during the 12 years. Seven patients were hospitalised for this reason. In most cases, infection $(n=5)$ led to admittance. In one case a failure of an insulin pump was the underlying reason, and one patient was hospitalised 20 times for approximately 159 days between 1994 and 1997 in various hospitals because of recurrent refusal to administer insulin. 


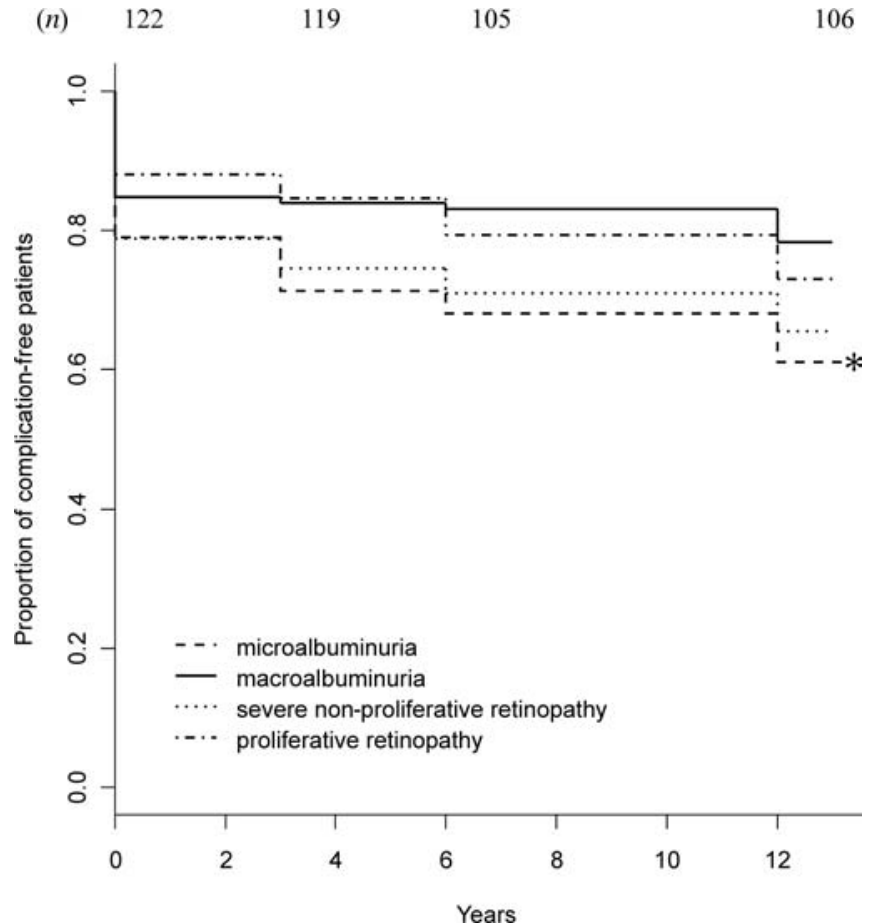

Fig. 2. Time to event curves for occurrence of complications. * Patients with current assessments of retinopathy and nephropathy. One patient did not provide us with urine samples and reports of ophthalmological examinations over the whole observational period, and a further two patients did not provide the data at 12-year follow-up

Insulin treatment and self-monitoring. At the 12-year follow-up, 103 (94\%) of 109 patients carried out IIT. Results of insulin treatment and self-monitoring are summarised in Table 2. Diabetes-related knowledge was graded at $46 \%$ (percentage of correct answers) before participation in the DTTP and reached $77 \%$ at the end of the teaching sessions. Without interim teaching, the percentage dropped to $70 \%$ and $64 \%$ after 3 and 6 years respectively. For optimisation of insufficient metabolic control, 18 patients participated in the DTTP after 6 years. After 12 years, overall knowledge was graded at $70 \%$.

After the 6-year re-evaluation, 33 patients decided to use short-acting insulin analogues, four patients decided to use long-acting insulin analogues, and ten patients switched from multiple injection to continuous insulin infusion therapy.

Complications and mortality. During the follow-up period, 11 patients died. In all cases, death was due to cardiovascular events. All but two patients had either an overt diabetic nephropathy (seven) at baseline or had developed macroalbuminuria (two) during followup. Over the 12-year period, the incidence of microalbuminuria was $21 \%$, the incidence of macroalbuminuria was $7 \%$, and the incidence of both severe nonproliferative and proliferative retinopathy was $16 \%$ (Fig. 2). Blood pressure remained relatively un-

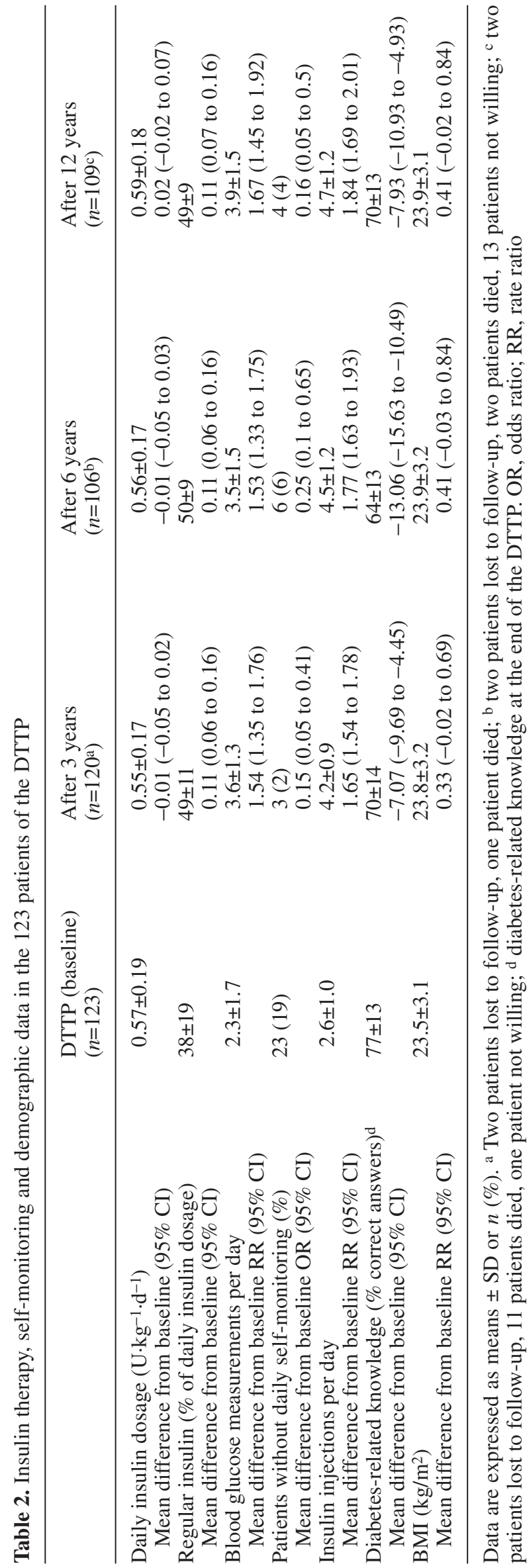


changed (baseline 131 $\pm 20 / 81 \pm 12 \mathrm{~mm} \mathrm{Hg}$; after 12 years $134 \pm 19 / 79 \pm 11 \mathrm{~mm} \mathrm{Hg}$ ), while antihypertensive prescriptions increased from in $21(17 \%)$ patients at baseline to in 51 (47\%) after 12 years.

The need for hospitalisation for acute metabolic disturbances (hypoglycaemia, hyperglycaemia or ketoacidosis) decreased from $4.5 \pm 11.2$ to $1.3 \pm 7.0,1.9 \pm 5.8$ and $0.6 \pm 2.8$ days per patient per year after 3,6 and 12 years respectively.

\section{Discussion}

In our cohort study, the initiation of IIT within the DTTP led to a persistent reduction in the frequency of severe hypoglycaemia and the number of patients who experienced such an episode. In addition, an improvement in metabolic control was observed after 3 years, although mean $\mathrm{HbA}_{1} \mathrm{c}$ values returned to baseline levels after 6 and 12 years. Approximately every other participant was able to improve metabolic control without reporting an episode of severe hypoglycaemia at any of the follow-up examinations. Good adherence to the IIT, indicated by a sustained increase in the number of daily insulin injections and blood glucose testing was also observed.

The effectiveness of teaching and treatment programmes for IIT has been studied extensively in randomised controlled trials $[7,10]$ and cohort studies [5, $9,11]$. The corroborating results obtained in different healthcare systems, e.g. significant and lasting reduction in glycosylated haemoglobin, no increase in severe hypoglycaemic episodes and reduction of hospital days, led us to implement this procedure in routine care. Therefore, this 12-year study lacks a control group, which is considered unethical. However, the changes experienced by these patients add to the evidence currently available with respect to patients on IIT for more than 10 years in a European outpatient healthcare setting. One large-scale cohort trial investigated prognostic factors related to diabetic late complications and mortality after 10 years $[15,16]$. In a recently repeated cross-sectional survey of Type 1 diabetic outpatients in Sweden, $\mathrm{HbA}_{1}$ c levels improved only marginally from $7.6 \%$ to $7.4 \%$ over 14 years with increasing use of intensified therapy, but the prevalence of severe hypoglycaemia increased from $17 \%$ to $27 \%$ [17], which is consistent with previous findings $[2,4,18]$.

While our results are suggestive of a long-standing effect of reducing severe hypoglycaemia, some matters on metabolic control must be regarded with caution. After initial significant improvement, substantial deterioration of metabolic control was observed after 6 years. This pattern is similar to results of a 6-year follow-up investigation of approximately 600 Type 1 diabetic patients [6] whose metabolic control worsened after 3 years. In contrast to the DCCT, where the intensified therapy group attended monthly visits at the centre and had even more telephone contact to review and adjust regimens, patients in our cohort were seen, on average, once to twice a year in our outpatient clinic. No differences in outcome were observed between patients who were in regular care (at least once a year) at our outpatient clinic and patients who were not. Therefore, a possible explanation is that intensification of insulin therapy without reinforcement and continuing support may not provide a prolonged distinctive improvement as demonstrated in the DCCT $[2,3]$. Moreover, since avoiding episodes of severe hypoglycaemia was one of the key priorities of the DTTP, some patients may have accepted higher glucose levels rather than risking severe hypoglycaemia. Patients' ability to perceive episodes of glucose dysregulation, and balancing this risk : benefit ratio could therefore be attributed to the structured training intervention programme, even though metabolic control is impaired for the benefit of fewer hypoglycaemic episodes [19]. However, less exposure to chronically elevated plasma glucose levels for a few years after the DTTP could be of overall biological significance in terms of the following points, as demonstrated by the EDIC study [3].

It is important to emphasise that psychological factors, such as active coping, social support and perception of self-efficacy, which are important for successful self-management of diabetes and were not routinely assessed in our investigation, had a great impact on metabolic control over the observational period [20, $21,22]$. Interim teaching in DTTP patients with insufficient metabolic control did not have an impact on outcomes at follow-up (data on file). Thus, to maintain acquired skills and psychological support, different models for teaching patients and for behavioural interventions need to be implemented [23].

One can only hypothesise as to whether a combination of short- and long-acting insulin analogues [24], devices for continuous glucose measurements [25] and/or islet transplantation [26] will help to improve metabolic control in Type 1 diabetic patients in the long run.

As observed in several earlier investigations, the presence of overt nephropathy was associated with excess mortality [15, 27, 28].

In conclusion, our investigation has shown that the initiation of IIT within a structured outpatient DTTP was followed, in the long term, by a reduced risk of severe hypoglycaemia in Type 1 diabetic patients. Initial improvements in metabolic control were not longstanding. However, in the long run, almost every second participant of the DTTP was able to improve metabolic control without an increase in the number of severe hypoglycaemic episodes. Most patients adhered to the complex intervention of IIT over the 12-year period. Therefore, a structured teaching and treatment programme should be an important consid- 
eration during the initiation of intensified insulin treatment.

Acknowledgements. We thank Gerhard Neubauer for statistical advice and Clara Horvath for reviewing the manuscript.

\section{References}

1. Bradley C, Speight J (2002) Patients perceptions of diabetes and diabetes therapy: assessing quality of life. Diabet Metab Res Rev 18 [Suppl 3]:64-69

2. The DCCT Research Group (1993) The effect of intensive treatment of diabetes on the development and progression of long-term complications in insulin-dependent diabetes mellitus. N Engl J Med 329:977-986

3. The Writing Team for the Diabetes Control and Complications Trial/Epidemiology of Diabetes Interventions and Complications Research Group (2002) Effect of intensive therapy on the microvascular complications of Type 1 diabetes mellitus. JAMA 287:2563-2569

4. The DCCT Research Group (1997) Hypoglycemia in the Diabetes Control and Complications Trial. Diabetes 46:271-286

5. Pieber TR, Brunner GA, Schnedl WJ, Schattenberg S, Kaufmann P, Krejs GJ (1995) Evaluation of a structured outpatient group education program for intensive insulin therapy. Diabetes Care 18:625-630

6. Bott S, Bott U, Berger M, Mühlhauser I (1997) Intensified insulin therapy and the risk of severe hypoglycaemia. Diabetologia 40:926-932

7. DAFNE Study Group (2002) Training in flexible, intensive insulin management to enable dietary freedom in people with Type 1 diabetes: dose adjustment for normal eating (DAFNE) randomised controlled trial. BMJ 325:746-751

8. Semlitsch B, Goritschan D, Zapotoczky H, Brunner GA, Siebenhofer A, Pieber TR (1997) Long term effect of a structured outpatient education programme in IDDMa 6 year follow-up. Diabetologia 40:A609 (Abstract)

9. Mühlhauser I, Jörgens V, Berger M et al. (1983) Bicentric evaluation of a teaching and treatment programme for Type 1 (insulin-dependent) diabetic patients: improvement of metabolic control and other measures of diabetes care for up to 22 months. Diabetologia 25:470-476

10. Mühlhauser I, Bruckner I, Berger M et al. (1987) Evaluation of an intensified insulin treatment programme as routine management of Type 1 (insulin-dependent) diabetes: the Bucharest-Düsseldorf Study. Diabetologia 30:681-690

11. Mühlhauser I, Berger M (2002) Patient education-evaluation of a complex intervention. Diabetologia 45:1723-1733

12. Halwachs-Baumann G, Katzensteiner S, Schnedl W, Pürstner P, Pieber TR, Wilders-Truschnig M (1997) Comparative evaluation of three assay systems for automated determination of hemoglobin $\mathrm{A}_{1 \mathrm{c}}$. Clinical Chemistry 43:511-517
13. Hardin JW, Hilbe JM (2003) Generalized estimating equations. Chapman \& HALL/CRC, London

14. Ihaka R, Gentleman DR (1996) A language for data analysis and graphics. J Comput Graph Stat 5:299-314

15. Mühlhauser I, Sawicki PT, Blank M, Overmann H, Bender R, Berger M (2000) Prognosis of persons with type 1 diabetes on intensified insulin therapy in relation to nephropathy. J Int Med 248:333-341

16. Mühlhauser I, Overmann H, Bender R, Jörgens V, Berger M (2000) Predictor of mortality and endstage diabetic complications in patients with Type 1 diabetes mellitus on intensified insulin therapy. Diabet Med 17:727-734

17. Bragd J, Adamson U, Lins PE, Wredling R, Oskarsson P (2003) A repeated cross-sectional survey of severe hypoglycaemia in 178 Type 1 diabetes mellitus patients performed in 1984 and 1998. Diabet Med 20:216-219

18. Reichard P, Nilsson BY, Rosenquist U (1993) The effect of long-term intensified insulin treatment on the development of microvascular complications of diabetes mellitus. N Engl J Med 329:304-309

19. Marrero DG, Guare JC, Vandagriff JL, Fineberg NS (1997) Fear of hypoglycemia in the parents of children and adolescents with diabetes: maladaptive or healthy response? Diabetes Educ 23:281-286

20. Rubin RR, Peyrot M (1992) Psycholosocial problems and interventions in diabetes: a review of the literature. Diabetes Care 15:1640-1657

21. Cox DJ, Gonder-Frederick L (1992) Major developments in behavioural diabetes research. J Consult Clin Psychol 60:628-638

22. Bott U, Jörgens V, Grüsser M, Bender R, Mühlhauser I, Berger M (1994) Predictors of glycaemic control in Type 1 diabetic patients after participation in an intensified treatment and teaching programme. Diabet Med 11:362-171

23. Hampson SE, Skinner TC, Hart J et al. (2000) Behavioral interventions for adolescents with type 1 diabetes. Diabetes Care 23:1416-1422

24. Hermansen K, Fontaine P, Kukolja KK, Peterkova V, Leth G, Galli MA (2004) Insulin analogues (insulin detemir and insulin aspart) versus traditional human insulins (NPH and regular human insulin) in basal-bolus therapy for patients with type 1 diabetes. Diabetologia 47:622-629

25. Garg SK, Schwartz S, Edelman SV (2004) Improved glucose excursions using an implantable real-time continuous glucose sensor in adults with type 1 diabetes. Diabetes Care 27:734-738

26. Robertson RP (2004) Islet transplantation as a treatment for diabetes-a work in progress. N Engl J Med 350:694705

27. Borch-Johnsen K, Andersen PK, Deckert T (1985) The effect of proteinuria on relative mortality in Type 1 (insulindependent) diabetes mellitus. Diabetologia 28:590-596

28. Tuomilehto J, Borch-Johnsen K, Molarius A (1998) Incidence of cardiovascular disease in Type 1 (insulin-dependent) diabetic subjects with and without diabetic nephropathy in Finland. Diabetologia 41:784-790 